\title{
Dental Students in Germany throughout the COVID-19 Pandemic: A Psychological Assessment and Cross-Sectional Survey
}

\author{
Mohamed Mekhemar ${ }^{1, * \mathbb{D}}$, Sameh Attia ${ }^{2} \mathbb{D}$, Christof Dörfer $^{1}$ and Jonas Conrad ${ }^{1, * \mathbb{D}}$ \\ 1 Clinic for Conservative Dentistry and Periodontology, School of Dental Medicine, Kiel University, \\ Arnold-Heller-Str. 3, Haus B, 24105 Kiel, Germany; doerfer@konspar.uni-kiel.de \\ 2 Department of Oral and Maxillofacial Surgery, Justus-Liebig University Giessen, Klinik Str. 33, \\ 35392 Giessen, Germany; sameh.attia@dentist.med.uni-giessen.de \\ * Correspondence: mekhemar@konspar.uni-kiel.de (M.M.); conrad@konspar.uni-kiel.de (J.C.); \\ Tel.: +49-431-597-2817 (M.M. \& J.C.)
}

Citation: Mekhemar, M.; Attia, S.; Dörfer, C.; Conrad, J. Dental Students in Germany throughout the COVID-19 Pandemic: A

Psychological Assessment and Cross-Sectional Survey. Biology 2021, 10, 611. https://doi.org/10.3390/ biology10070611

Academic Editors:

Mohamad Goldust, Robert

A. Schwartz, Dedee Murrell, Torello Lotti and Tetsuya Shiuchi

Received: 2 June 2021

Accepted: 29 June 2021

Published: 1 July 2021

Publisher's Note: MDPI stays neutral with regard to jurisdictional claims in published maps and institutional affiliations.

Copyright: (c) 2021 by the authors. Licensee MDPI, Basel, Switzerland. This article is an open access article distributed under the terms and conditions of the Creative Commons Attribution (CC BY) license (https:/ / creativecommons.org/licenses/by/ $4.0 /)$.
Simple Summary: The Coronavirus Disease (COVID-19) outbreak has presented severe public health risks throughout the world since 2019. Among various health impacts of the pandemic globally, it has led to unprecedented risks to the mental wellbeing of healthcare professionals and students. This study aimed to examine the impact of the pandemic and several demographic and social factors on dental students' mental health in German universities nationwide from July 2020 to January 2021. The survey assembled data through an online platform on demographics, the Depression Anxiety Stress Scales (DASS-21), and the Impact of Events Scale-Revised (IES-R) instrument from 211 participants and examined correlations between all variables and mental consequences of depression, anxiety, stress, intrusion, avoidance, and hyperarousal in the study population. Dental students showed an overall mild impact of the outbreak on the assessed psychological aspects. Female students and students having cardiovascular conditions or smoking habitually, as well as participants perceiving COVID-19 as a financial danger were significantly affected more by the disease than their counterparts. Highlighting these results can help to protect dental students' mental health in German universities during the crisis and support the authorities to adjust the required actions to counteract detrimental effects of the outbreak

Abstract: Multiple investigations have reported high psychological distress among students since the coronavirus (COVID-19) outbreak started. This survey examined the associations between psychological features, and several demographic and social factors among dental students in German universities. Dental students registered in German universities nationwide were asked to join this survey via a self-directed online questionnaire, from July 2020 to January 2021. This study assembled data on demographic statistics, the depression anxiety stress scales (DASS-21), and the impact of events scale-revised (IES-R) instrument. The relationships between demographic-related variables and mental consequences of depression, anxiety, stress, intrusion, avoidance, and hyperarousal were inspected. Two hundred and eleven students contributed to the questionnaire and conveyed overall normal or mild outcomes of depression, anxiety, stress, intrusion, avoidance, and hyperarousal. In addition, female gender, cardiovascular diseases, smoking habits, and seeing the COVID-19 outbreak as a financial risk were stated as significant related factors $(p<0.05)$, with increased IES-R and DASS-21 scores. These results highlight the features that should be considered to better protect dental students' mental health in German universities during the crisis.

Keywords: COVID-19; IES-R; DASS-21; anxiety; stress; depression; dental students; psychological impact; mental health 


\section{Introduction}

In December 2019, the city of Wuhan in China became the epicenter of a pneumonia epidemic of uncertain origin, later recognized as SARS-CoV-2 and named, by the World Health Organization, as the 2019 novel coronavirus (COVID-19). Because of its high virulence and mortality, this strain was declared a worldwide pandemic on March 11 2020. Its increased infection rate, along with a rising number of associated severe health deterioration or deaths, sparked intense public unrest and panic. Early research on the immediate mental outcomes of the current outbreak on the general population identified mild to extreme psychological consequences of the disease [1,2].

In addition to its psychological impact on the general population, the COVID-19 pandemic has challenged and often surpassed the capacity of health professionals and facilities worldwide. Healthcare workers had to continue providing care for patients in spite of physical and mental exhaustion, high risk of infection, their fear to transmit the disease to their loved ones, and the loss of many patients. Additionally, long working shifts combined with heavy restrictions, together with personal isolation, have affected the mental status of various health professionals [3]. Similar to other medical branches, the COVID-19 outbreak hindered the efforts of dental healthcare professionals and students [4,5]. Multiple dental procedures have been suspended due to the increased risk of viral cross-infection during treatment, limiting dental visits only to emergency cases [6].

Dental manufacturers and several dental practices have suspended some employees, as an outcome of the financial pressure during the pandemic [7]. In fact, dentistry was classified among other medical branches as a high-risk profession for the likely occupational virus transmission, due to the close proximity of dental healthcare personnel to the patient throughout the treatment, and the procedural generation of infective aerosols [8]. Dentistry students, in specific, are considered at a higher risk than all other dental healthcare workers for COVID-19 infection, owing to their inexperience, practical insufficiency, and deficiency of knowledge [9]. Besides their worries about cross-infection among their patients and families, the social implications of lockdowns and the discontinuing or rapid changes in scientific or educational programs might present effective sources of mental distress among the students $[4,6,9]$.

In Germany, the current pandemic has resulted in over one million cases of COVID-19 infections, and multiple deaths. Though COVID-19-related deaths are relatively uncommon in Germany's younger age groups, they account for a significant portion of COVID-19related hospitalizations [10-12]. Furthermore, during the first wave of outbreaks in 2020, researchers discovered that people between 20 and 24 years of age play a key role in spreading the SARS-CoV-2 infection in Germany [13]. This implies that university students, the majority of whom are in this age group, may play a crucial role in SARS-CoV-2 transmission [12]. Furthermore, during the second wave of infection in the summer and autumn of 2020, individuals of the student group were found to be more prevalent among those infected, confirming the importance of observing and understanding this group and its circumstances during the pandemic [12]. With about 80.5 million people, almost 70,000 dentists, and 32 dental schools, Germany, the most populous nation of the European Union, is among the largest European states in terms of the number of oral health professionals and students [14,15]. In recent years, the mental wellbeing of dental and medical students, particularly during difficult situations, has been a growing concern worldwide, with an estimation of potential mental disorders in one in every five students [16]. Although earlier investigations reported the psychological distress affecting German dentists throughout the outbreak [6], the psychological consequences on dental students, as one of the main dental service providers in university clinics and future dental professionals [15], accompanying the crisis and its key aspects, still need to be disclosed. Thus, this investigation intended to explore this subject via the impact of event scale-Revised (IES-R) and depression anxiety stress scale (DASS-21) examinations on a national scale among Germany's dental students. 


\section{Materials and Methods}

\subsection{Study Population and Procedures}

A national cross-sectional study of dental students in Germany was conducted to assess the psychological outcome of COVID-19 and its associated causes. An online survey was developed using an internet-based survey platform to reduce face-to-face communication and encourage contribution by all students (Unipark, QuestBack $\mathrm{GmbH}$, Cologne, Germany). Following the University of Kiel Ethics Board (D452/18) approval, a link for the online survey was posted on numerous social network pages associated with dentistry and invitations were sent to registered dental students from various universities in Germany, as performed in previous studies [15]. The survey's introductory text quickly clarified the study and the voluntary and anonymous involvement of the students. There were no financial benefits assured to participating students, and no exclusion requirements were established (e.g., gender, age, or nationality). At the start of the survey, students consented, indicating their willingness to participate. Data collection was completed from July 2020 to January 2021.

\subsection{Survey Instruments}

The primary segment of the survey gathered sociodemographic data from respondents such as their age, federal state, parenthood, marital status, systemic comorbidities, and smoking status. Students also stated if they believed the outbreak represented a private financial risk.

Following up on that point, in the second portion of the questionnaire, participants received the depression anxiety stress scale (DASS-21) and impact of event scale-revised (IES-R) tool. Both survey instruments were applied with their scoring systems and cutoff scores to evaluate depression, anxiety, stress, as well as intrusion, avoidance, and hyperarousal as applied for German dentists previously [6]. The DASS-21 subscales were scored as follows: normal (0-4 DASS-21 points), mild (5-6 DASS-21 points), moderate (7-10 DASS-21 points), severe (11-13 DASS-21 points), and extremely severe (14+ DASS-21 points) for depression; normal (0-3 DASS-21 points), mild (4-5 DASS-21 points), moderate (6-7 DASS-21 points), severe (8-9 DASS-21 points), and extremely severe (10+ DASS-21 points) for anxiety; and normal (0-7 DASS-21 points), mild (8-9 DASS-21 points), moderate (10-12 DASS-21 points), severe (13-16 DASS-21 points), and extremely severe (17+ DASS-21 points) for stress. The IES-R subscores were categorized as normal (0-23 IES-R points), mild (24-32 IES-R points), moderate (33-36 IES-R points), and severe psychological impact of events ( $>37$ IES-R points). Both survey instruments were previously validated in their German version and displayed a significant reliability to measure the psychological impact of the COVID-19 pandemic on general populations and healthcare personnel [6].

\subsection{Sample Size Calculation}

To decide how many responses were required for a significant size of the sample population, conditions were defined analogously to earlier investigations on dental students in Germany [15] for the sample size calculation.

The following conditions were defined:

1. Number of sent emails to registered students $(N=207)$;

2. A confidence level of $95 \%$;

3. A margin of error of $5 \%$.

Based on this calculation, it was decided that at least 135 dental students were required for a sufficient study group across Germany.

\subsection{Statistical Analysis}

Outcomes were digitally recorded by the online-based survey instrument and analyzed afterwards by SPSS software (SPSS Statistic 27, IBM, Armonk, NY, USA). Descriptive analysis was completed and the normality of all data variables was tested by the Kolmogorov and Shapiro-Wilk test. Data of all scales and sub-scales showed no normal 
distribution. No transformations of variables were attempted for normalization based on the statistical analysis performed previously on German dentists [6]. Skewed distributions of variables have also been reported to be commonly used and accepted in psychological and social research and do not require normalization procedures necessarily $[17,18]$. To examine the relations between DASS-21/IES-R ratings and sociodemographic factors, univariate analyses (Kruskal-Wallis and Mann-Whitney $U$ test) were applied. For significant statistical test outcomes post hoc, the Dunn-Bonferroni statistical test was further applied in single comparisons. Then multiple linear regression tests were executed on DASS-21 total and subscores and the IES-R subscales to distinguish the involvement of the earlier recognized, relevant factors. Statistical significance was set at $p<0.05$.

\section{Results}

\subsection{Participation and Sociodemographic Data}

An overall number of 211 dental students contributed to the study, displaying a sample size that was sufficient for the required statistical power. The students encompassed female $(73.5 \%)$, male $(26 \%)$, and third-gender dental students $(0.5 \%)$ from all dental schools in Germany. All of the respondents were younger than 50 years old. Most of the participating students were single $(81.5 \%)$ and did not have any children $(96.7 \%)$, while the rest were either in a marriage or marriage-like relationship $(18.5 \%)$, and were responsible for children (3.3\%). Around one-third of the participants (32.7\%) considered the COVID-19 outbreak to be a danger to their financial stability. Additionally, the study group exhibited a $5.7 \%$ rate of smoking and displayed multiple medical comorbidities, with $3.3 \%$ cardiovascular diseases, $2.4 \%$ chronic pulmonary conditions, and $0.9 \%$ immunodeficiencies (Table 1 ).

Table 1. Characteristics of participants $(n=211)$.

\begin{tabular}{|c|c|c|c|}
\hline Sociodemographic Factors & & $\mathbf{N}$ & $\%$ \\
\hline \multicolumn{4}{|l|}{ Gender } \\
\hline & Female & 155 & 73.5 \\
\hline & Male & 55 & 26.0 \\
\hline & Third Gender & 1 & 0.5 \\
\hline \multicolumn{4}{|l|}{ Age } \\
\hline & $18-49$ & 211 & 100.0 \\
\hline & $50-59$ & 0 & 0 \\
\hline & $\geq 60$ & 0 & 0 \\
\hline \multicolumn{4}{|l|}{ Marital status } \\
\hline & Single & 172 & 81.5 \\
\hline & Married or in a marriage-like partnership & 39 & 18.5 \\
\hline & Divorced, separated, or widowed & 0 & 0 \\
\hline \multicolumn{4}{|l|}{ Having children } \\
\hline & Yes & 7 & 3.3 \\
\hline & No & 204 & 96.7 \\
\hline \multicolumn{4}{|c|}{ COVID-19 being a personal financial threat } \\
\hline & Yes & 69 & 32.7 \\
\hline & No & 142 & 67.3 \\
\hline \multicolumn{4}{|l|}{ Smoker } \\
\hline & Yes & 12 & 5.7 \\
\hline & No & 199 & 94.3 \\
\hline
\end{tabular}


Table 1. Cont.

\begin{tabular}{llll}
\hline Medical comorbidity $^{\mathbf{1}}$ & & \\
\hline & $\begin{array}{l}\text { Diseases of the cardiovascular system (e.g., } \\
\text { coronary heart disease and high blood pressure) }\end{array}$ & 7 & 3.3 \\
\hline Chronic lung diseases (e.g., COPD) & 5 & 2.4 \\
\hline Chronic liver diseases & 0 & 0 \\
\hline Diabetes mellitus & 0 & 0 \\
\hline Cancer & 0 & 0 \\
\hline Immunodeficiency & 2 & 0.9 \\
\hline
\end{tabular}

${ }^{1}$ No or multiple choice was possible.

\subsection{DASS-21 and IES-R Scales and Associated Factors}

The inspection of psychological indicators in the study sample, by the DASS-21 and IES-R scales, are displayed in Tables 2 and 3, and in relation to the associated aspects in Tables 4 and 5, respectively.

Table 2. DASS-21 and IES-R scores of the study sample.

\begin{tabular}{llll}
\hline \multicolumn{1}{l}{ Psychological Evaluation } & & Mean \pm SD & Interquartile Range \\
\hline DASS-21 $(\boldsymbol{n = 2 1 1})^{\mathbf{1}}$ & & & \\
\hline & Total & $11.74 \pm 10.03$ & 13 \\
\hline & Depression & $4.01 \pm 3.93$ & 5 \\
\hline & Anxiety & $2.52 \pm 3.20$ & 4 \\
\hline IES-R $\left(\boldsymbol{n = 2 0 4 )} \mathbf{1}^{\mathbf{1}}\right.$ & Stress & $5.21 \pm 4.09$ & 6 \\
\hline & & & 7 \\
\hline & Intrusion & $5.27 \pm 5.54$ & 11 \\
\hline & Avoidance & $7.74 \pm 7.40$ & 9 \\
\hline
\end{tabular}

${ }^{1} n$ varies because of missing data.

The applied grading system exhibited that the total survey population of dental students displayed DASS-21 and IES-R scores of normal psychological behaviors, with potential slight distress due to the outbreak (Tables 2 and 3).

The DASS-21 and IES-R scales-related factors presented statistically higher DASS-21, and IES-R total and subscale outcomes, labelling mild to normal mental consequences amongst dental students of the female or third gender, having cardiovascular diseases, with a smoking habit, as well as students seeing the outbreak as a financial danger (Tables 4 and 5).

Multiple regression analyses of DASS-21 total and subscores within the study model showed a significant impact of financial factors, systemic cardiovascular diseases, the participants 'gender, and habitual smoking on the psychological stress, depression, and anxiety, as well as intrusion, avoidance, and hyperarousal of German dental students (Tables 6 and 7). 
Table 3. The number of dental students and total population percentage for each DASS-21 and IES-R subscale category.

\begin{tabular}{|c|c|c|c|}
\hline Subscale & Category & $\mathbf{N}$ & $\%$ \\
\hline \multicolumn{4}{|l|}{ DASS-21 $(n=211)^{1}$} \\
\hline Depression & normal & 134 & 63.5 \\
\hline & mild & 37 & 17.5 \\
\hline & moderate & 24 & 11.4 \\
\hline & severe & 9 & 4.3 \\
\hline & extremely severe & 7 & 3.3 \\
\hline Anxiety & normal & 155 & 73.5 \\
\hline & mild & 26 & 12.3 \\
\hline & moderate & 11 & 5.2 \\
\hline & severe & 9 & 4.3 \\
\hline & extremely severe & 10 & 4.7 \\
\hline Stress & normal & 157 & 74.4 \\
\hline & mild & 21 & 10.0 \\
\hline & moderate & 20 & 9.5 \\
\hline & severe & 10 & 4.7 \\
\hline & extremely severe & 3 & 1.4 \\
\hline \multicolumn{4}{|l|}{ IES-R $(n=204)^{1}$} \\
\hline Intrusion & normal & 204 & 100.0 \\
\hline & mild & 0 & 0 \\
\hline & moderate & 0 & 0 \\
\hline & severe & 0 & 0 \\
\hline Avoidance & normal & 196 & 96.1 \\
\hline & mild & 6 & 2.9 \\
\hline & moderate & 2 & 1.0 \\
\hline & severe & 0 & 0 \\
\hline Hyperarousal & normal & 202 & 95.7 \\
\hline & mild & 2 & 0.9 \\
\hline & moderate & 0 & 0 \\
\hline & severe & 0 & 0 \\
\hline
\end{tabular}

\footnotetext{
${ }^{1} n$ varies because of missing data.
} 
Table 4. Differences between participants' characteristics regarding DASS-21 total and subscale scores.

\begin{tabular}{|c|c|c|c|c|c|c|c|c|c|c|c|c|}
\hline & \multicolumn{3}{|c|}{ DASS-21 Total } & \multicolumn{3}{|c|}{ DASS-21 Depression } & \multicolumn{3}{|c|}{ DASS-21 Anxiety } & \multicolumn{3}{|c|}{ DASS-21 Stress } \\
\hline & Mean \pm SD & Test Statistic & $p$-Value & Mean \pm SD & Test Statistic & $p$-Value & Mean \pm SD & Test Statistic & $p$-Value & Mean \pm SD & Test Statistic & $p$-Value \\
\hline \multicolumn{13}{|l|}{ Gender } \\
\hline Female & $12.57 \pm 9.94$ & \multirow{3}{*}{$\mathrm{H}=10.81$} & \multirow{3}{*}{$<0.01$} & $4.19 \pm 3.76$ & \multirow{3}{*}{$\mathrm{H}=8.08$} & \multirow{3}{*}{0.02} & $2.75 \pm 3.26$ & \multirow{3}{*}{$\mathrm{H}=10.64$} & \multirow{3}{*}{0.01} & $5.63 \pm 4.08$ & \multirow{3}{*}{$\mathrm{H}=11.22$} & \multirow{3}{*}{$<0.01$} \\
\hline Male & $8.82 \pm 8.91$ & & & $3.31 \pm 4.05$ & & & $1.67 \pm 2.67$ & & & $3.84 \pm 3.62$ & & \\
\hline Third Gender & $44.00 \pm 0.00$ & & & $16.00 \pm 0.00$ & & & $12.00 \pm 0.00$ & & & $16.00 \pm 0.00$ & & \\
\hline \multicolumn{13}{|l|}{ Post hoc: Dunn-Bonferroni Test } \\
\hline Male-Third G & & -122.47 & 0.05 & & -117.17 & 0.06 & & -119.78 & 0.05 & & -122.89 & 0.05 \\
\hline Female-Third G & & -95.39 & 0.12 & & -95.23 & 0.12 & & -93.63 & 0.12 & & -95.25 & 0.12 \\
\hline \multicolumn{13}{|l|}{ Age } \\
\hline $18-49$ & $11.74 \pm 10.03$ & & \multirow{3}{*}{-} & $4.01 \pm 3.93$ & & \multirow{3}{*}{-} & $2.52 \pm 3.20$ & & \multirow{3}{*}{-} & $5.21 \pm 4.09$ & & \multirow{3}{*}{-} \\
\hline $50-59$ & - & - & & - & - & & - & - & & - & - & \\
\hline$\geq 60$ & - & & & - & & & - & & & - & & \\
\hline \multicolumn{13}{|l|}{ Marital status } \\
\hline $\begin{array}{l}\text { Married or in a } \\
\text { marriage-like partnership }\end{array}$ & $12.82 \pm 10.41$ & \multirow[t]{2}{*}{$\mathrm{H}=0.50$} & \multirow[t]{2}{*}{0.48} & $4.15 \pm 3.49$ & \multirow[t]{2}{*}{$\mathrm{H}=0.58$} & \multirow[t]{2}{*}{0.45} & $3.08 \pm 4.06$ & \multirow[t]{2}{*}{$\mathrm{H}=0.24$} & \multirow[t]{2}{*}{0.62} & $5.59 \pm 4.00$ & \multirow[t]{2}{*}{$\mathrm{H}=0.56$} & \multirow[t]{2}{*}{0.46} \\
\hline Divorced, separated, or widowed & - & & & - & & & - & & & - & & \\
\hline \multicolumn{13}{|l|}{ Having children } \\
\hline Yes & $16.57 \pm 14.54$ & $U-58650$ & 042 & $6.29 \pm 6.05$ & $J-56450$ & 0.34 & $4.43 \pm 4.72$ & U - 54400 & & $5.86 \pm 4.60$ & & \\
\hline No & $11.58 \pm 9.85$ & $0=300.30$ & 0.42 & $3.94 \pm 3.83$ & $0=504.50$ & & $2.45 \pm 3.14$ & $0=544.00$ & 0.27 & $5.19 \pm 4.08$ & $U=653.00$ & 0.70 \\
\hline COVID-19 being a personal finan & al threat & & & & & & & & & & & \\
\hline Yes & $13.71 \pm 10.76$ & U - 407350 & 005 & $4.86 \pm 4.46$ & $U-406550$ & 004 & $2.99 \pm 3.39$ & $U-425950$ & 012 & $5.87 \pm 4.05$ & $U-412250$ & 006 \\
\hline No & $10.79 \pm 59.55$ & $0=40 / 0.00$ & 0.00 & $3.61 \pm 3.59$ & $0=4000.50$ & 0.04 & $2.29 \pm 3.09$ & $0=4209.50$ & 0.12 & $4.89 \pm 4.09$ & $U=412<.00$ & 0.00 \\
\hline Smoker & & & & & & & & & & & & \\
\hline Yes & $18.83 \pm 13.74$ & & & $6.92 \pm 5.57$ & & & $4.25 \pm 5.07$ & & & $7.67 \pm 4.16$ & & \\
\hline No & $11.32 \pm 9.64$ & $U=7 / 5.50$ & 0.04 & $3.84 \pm 3.75$ & $U=762.50$ & 0.03 & $2.41 \pm 3.04$ & $U=894.50$ & 0.14 & $5.07 \pm 4.05$ & $U=761.50$ & 0.03 \\
\hline
\end{tabular}


Table 4. Cont.

\begin{tabular}{|c|c|c|c|c|c|c|c|c|c|c|c|c|}
\hline & \multicolumn{3}{|l|}{ DASS-21 Total } & \multicolumn{3}{|c|}{ DASS-21 Depression } & \multicolumn{3}{|c|}{ DASS-21 Anxiety } & \multicolumn{3}{|c|}{ DASS-21 Stress } \\
\hline & Mean \pm SD & Test Statistic & $p$-Value & Mean \pm SD & Test Statistic & $p$-Value & Mean \pm SD & Test Statistic & $p$-Value & Mean \pm SD & Test Statistic & $p$-Value \\
\hline \multicolumn{13}{|c|}{ Medical comorbidity (Multiple Choice) } \\
\hline No medical comorbidities & $11.43 \pm 9.62$ & & & $3.92 \pm 3.78$ & & & $2.38 \pm 3.00$ & & & $5.13 \pm 4.04$ & & \\
\hline $\begin{array}{l}\text { Diseases of the cardiovascular } \\
\text { system (e.g., coronary heart } \\
\text { disease and high blood pressure) }\end{array}$ & $24.71 \pm 16.00$ & $\mathrm{U}=1084.00$ & 0.02 & $8.57 \pm 6.43$ & $\mathrm{U}=1038.00$ & 0.04 & $7.57 \pm 5.47$ & $\mathrm{U}=1140.50$ & 0.01 & $8.57 \pm 5.53$ & $\mathrm{U}=1000.50$ & 0.07 \\
\hline $\begin{array}{l}\text { Chronic lung diseases } \\
\text { (e.g., COPD) }\end{array}$ & $7.60 \pm 5.73$ & $\mathrm{U}=397.50$ & 0.38 & $2.40 \pm 1.95$ & $\mathrm{U}=410.00$ & 0.43 & $1.60 \pm 1.67$ & $\mathrm{U}=466.50$ & 0.71 & $3.60 \pm 2.97$ & $\mathrm{U}=408.00$ & 0.43 \\
\hline Chronic liver diseases & - & - & - & - & - & - & - & - & - & - & - & - \\
\hline Diabetes mellitus & - & - & - & - & - & - & - & - & - & - & - & - \\
\hline Cancer & - & - & - & - & - & - & - & - & - & - & - & - \\
\hline Immunodeficiency & $7.50 \pm 0.71$ & $\mathrm{U}=171.50$ & 0.66 & $1.50 \pm 0.71$ & $\mathrm{U}=132.00$ & 0.37 & $0.50 \pm 0.71$ & $\mathrm{U}=123.50$ & 0.31 & $5.50 \pm 0.71$ & $\mathrm{U}=237.00$ & 0.74 \\
\hline
\end{tabular}

$\mathrm{SD}=$ standard deviation; $\mathrm{H}$ = test statistic of Kruskal-Wallis test; $\mathrm{U}=$ test statistic of Mann-Whitney $\mathrm{U}$ test; significant results are highlighted.

Table 5. Differences between participants' characteristics regarding IES-R subscale scores.

\begin{tabular}{|c|c|c|c|c|c|c|c|c|c|}
\hline & \multicolumn{3}{|c|}{ IES-R Intrusion } & \multicolumn{3}{|c|}{ IES-R Avoidance } & \multicolumn{3}{|c|}{ IES-R Hyperarousal } \\
\hline & Mean \pm SD & Test Statistic & $p$-Value & Mean \pm SD & Test Statistic & $p$-Value & Mean \pm SD & Test Statistic & $p$-Value \\
\hline \multicolumn{10}{|l|}{ Gender } \\
\hline Male & $3.08 \pm 3.55$ & \multirow[t]{2}{*}{$\mathrm{H}=10.97$} & \multirow[t]{2}{*}{$<0.01$} & $5.37 \pm 6.71$ & \multirow[t]{2}{*}{$\mathrm{H}=9.54$} & \multirow[t]{2}{*}{$<0.01$} & $4.29 \pm 5.07$ & \multirow[t]{2}{*}{$\mathrm{H}=8.50$} & \multirow[t]{2}{*}{$<0.01$} \\
\hline Third Gender & - & & & - & & & - & & \\
\hline \multicolumn{10}{|l|}{ Age } \\
\hline $18-49$ & $5.27 \pm 5.54$ & & & $7.74 \pm 7.40$ & & & $6.21 \pm 6.07$ & & \\
\hline $50-59$ & - & & & - & & & - & & \\
\hline$\geq 60$ & - & & & - & & & - & & \\
\hline
\end{tabular}


Table 5. Cont.

\begin{tabular}{|c|c|c|c|c|c|c|c|c|c|}
\hline & \multicolumn{3}{|c|}{ IES-R Intrusion } & \multicolumn{3}{|c|}{ IES-R Avoidance } & \multicolumn{3}{|c|}{ IES-R Hyperarousal } \\
\hline & Mean \pm SD & Test Statistic & $p$-Value & Mean \pm SD & Test Statistic & $p$-Value & Mean \pm SD & Test Statistic & $p$-Value \\
\hline \multicolumn{10}{|l|}{ Marital status } \\
\hline Married or in a marriage-like partnership & $5.64 \pm 5.86$ & \multirow[t]{2}{*}{$\mathrm{H}=0.07$} & \multirow[t]{2}{*}{0.79} & $8.85 \pm 8.53$ & \multirow[t]{2}{*}{$\mathrm{H}=0.33$} & \multirow[t]{2}{*}{0.57} & $7.67 \pm 6.27$ & \multirow[t]{2}{*}{$\mathrm{H}=2.71$} & \multirow[t]{2}{*}{0.10} \\
\hline Divorced, separated, or widowed & - & & & - & & & - & & \\
\hline \multicolumn{10}{|l|}{ Having children } \\
\hline Yes & $6.29 \pm 5.65$ & \multirow{2}{*}{$\mathrm{U}=590.00$} & \multirow{2}{*}{0.51} & $10.14 \pm 9.53$ & \multirow{2}{*}{$\mathrm{U}=582.50$} & \multirow{2}{*}{0.48} & $8.43 \pm 7.23$ & \multirow{2}{*}{$\mathrm{U}=562.00$} & \multirow{2}{*}{0.40} \\
\hline No & $5.24 \pm 5.55$ & & & $7.65 \pm 7.33$ & & & $6.13 \pm 6.03$ & & \\
\hline \multicolumn{10}{|l|}{ COVID-19 being a personal financial threat } \\
\hline Yes & $6.21 \pm 5.45$ & \multirow{2}{*}{$\mathrm{U}=3692.00$} & \multirow{2}{*}{0.03} & $9.17 \pm 8.11$ & \multirow{2}{*}{$\mathrm{U}=3910.50$} & \multirow{2}{*}{0.10} & $8.20 \pm 6.30$ & \multirow{2}{*}{$\mathrm{U}=3166.50$} & \multirow{2}{*}{$<0.01$} \\
\hline No & $4.83 \pm 5.55$ & & & $7.05 \pm 6.96$ & & & $5.26 \pm 5.74$ & & \\
\hline \multicolumn{10}{|l|}{ Smoker } \\
\hline Yes & $6.67 \pm 7.80$ & $\mathrm{U}=1097.50$ & 0.78 & $10.00 \pm 9.82$ & $\mathrm{U}=1030.00$ & 0.54 & $12.00 \pm 8.33$ & $\mathrm{U}=641.00$ & 0.01 \\
\hline \multicolumn{10}{|l|}{ Medical comorbidity (Multiple Choice) } \\
\hline No medical comorbidities & $5.14 \pm 5.45$ & & & $7.68 \pm 7.38$ & & & $6.03 \pm 5.92$ & & \\
\hline $\begin{array}{l}\text { Diseases of the cardiovascular system (e.g., } \\
\text { coronary heart disease and high blood pressure) }\end{array}$ & $11.17 \pm 7.91$ & $\mathrm{U}=877.00$ & 0.05 & $13.67 \pm 9.00$ & $\mathrm{U}=850.50$ & 0.07 & $14.17 \pm 8.31$ & $\mathrm{U}=934.00$ & 0.02 \\
\hline Chronic lung diseases (e.g., COPD) & $4.00 \pm 3.24$ & $\mathrm{U}=481.50$ & 0.90 & $3.20 \pm 3.11$ & $\mathrm{U}=324.50$ & 0.18 & $4.40 \pm 4.04$ & $\mathrm{U}=435.50$ & 0.63 \\
\hline Chronic liver diseases & - & - & - & - & - & - & - & - & - \\
\hline Diabetes mellitus & - & - & - & - & - & - & - & - & - \\
\hline Cancer & - & - & - & - & - & - & - & - & \\
\hline Immunodeficiency & $4.00 \pm 1.41$ & $\mathrm{U}=212.00$ & 0.90 & $7.00 \pm 1.41$ & $\mathrm{U}=225.50$ & 0.78 & $4.00 \pm 0.00$ & $\mathrm{U}=193.00$ & 0.91 \\
\hline
\end{tabular}


Table 6. Multiple regression analyses with relevant factors of DASS-21 total and subscores.

\begin{tabular}{|c|c|c|c|c|c|c|}
\hline & B & SE & $\beta$ & $\mathbf{T}$ & $\mathbf{p}$ & $95 \% \mathrm{CI}$ \\
\hline \multicolumn{7}{|l|}{ DASS-21 Total } \\
\hline Gender $^{1}$ & -3.06 & 1.46 & -0.14 & -2.09 & 0.04 & $-5.93 ;-0.18$ \\
\hline COVID-19 being personal financial threat ${ }^{2}$ & -2.30 & 1.41 & -0.11 & -1.64 & 0.10 & $-5.07 ; 0.47$ \\
\hline Smoker $^{2}$ & -6.85 & 2.86 & -0.16 & -2.34 & 0.02 & $-12.47 ;-1.22$ \\
\hline $\begin{array}{l}\text { Medical comorbidity: diseases of the } \\
\text { cardiovascular system (e.g., coronary heart } \\
\text { disease and high blood pressure) }{ }^{3}\end{array}$ & 13.52 & 3.70 & 0.24 & 3.65 & $<0.01$ & $6.22 ; 20.81$ \\
\hline \multicolumn{7}{|l|}{ DASS-21 Depression } \\
\hline Gender $^{1}$ & -0.63 & 0.58 & -0.07 & -1.09 & 0.28 & $-1.77 ; 0.51$ \\
\hline COVID-19 being personal financial threat ${ }^{2}$ & -1.04 & 0.56 & -0.12 & -1.86 & -0.06 & $-2.13 ; 0.06$ \\
\hline Smoker $^{2}$ & -2.74 & 1.13 & -0.16 & -2.43 & 0.02 & $-4.97 ;-0.51$ \\
\hline $\begin{array}{l}\text { Medical comorbidity: diseases of the } \\
\text { cardiovascular system (e.g., coronary heart } \\
\text { disease and high blood pressure) }{ }^{3}\end{array}$ & 4.56 & 1.46 & 0.21 & 3.11 & $<0.01$ & $1.67 ; 7.44$ \\
\hline \multicolumn{7}{|l|}{ DASS-21 Anxiety } \\
\hline Gender $^{1}$ & -0.90 & 0.47 & -0.13 & -1.93 & 0.06 & $-1.81 ; 0.02$ \\
\hline $\begin{array}{l}\text { Medical comorbidity: diseases of the } \\
\text { cardiovascular system (e.g., coronary heart } \\
\text { disease and high blood pressure) }{ }^{3}\end{array}$ & 5.51 & 1.18 & 0.31 & 4.66 & $<0.01$ & $3.18 ; 7.84$ \\
\hline \multicolumn{7}{|l|}{ DASS-21 Stress } \\
\hline Gender $^{1}$ & -0.63 & 0.48 & -0.09 & -1.30 & 0.20 & $-1.58 ; 0.33$ \\
\hline
\end{tabular}

$\mathrm{B}=$ unstandardized beta coefficient; $\mathrm{SE}=$ standard error; $\beta=$ standardized beta coefficient; $p=p$-value; $\mathrm{CI}$ : confidence interval; significant results are highlighted; ${ }^{1} 1=$ female; $2=$ male; $3=$ third gender; ${ }^{2} 1=$ yes; $2=$ no; ${ }^{3} 0=$ not quoted; $1=$ quoted .

Table 7. Multiple regression analyses with relevant factors of IES-R scores.

\begin{tabular}{|c|c|c|c|c|c|c|}
\hline IES-R Intrusion & B & SE & $\beta$ & $\mathrm{T}$ & $\mathrm{p}$ & $95 \%$ CI \\
\hline Gender $^{1}$ & -2.92 & 0.86 & -0.23 & -3.41 & $<0.01$ & $-4.60 ;-1.23$ \\
\hline COVID-19 being personal financial threat ${ }^{2}$ & -1.13 & 0.80 & -0.10 & -1.42 & 0.16 & $-2.70 ; 0.44$ \\
\hline $\begin{array}{l}\text { Medical comorbidity: diseases of the } \\
\text { cardiovascular system (e.g., coronary heart } \\
\text { disease and high blood pressure })^{3}\end{array}$ & 6.30 & 2.20 & 0.19 & 2.87 & 0.01 & $1.97 ; 10.62$ \\
\hline \multicolumn{7}{|l|}{ IES-R Avoidance } \\
\hline Gender $^{1}$ & -3.18 & 1.17 & -0.19 & -2.72 & 0.01 & $-5.49 ;-0.87$ \\
\hline \multicolumn{7}{|l|}{ IES-R Hyperarousal } \\
\hline Gender $^{1}$ & -2.72 & 0.90 & -0.20 & -3.03 & $<0.01$ & $-4.50 ;-0.95$ \\
\hline COVID-19 being personal financial threat ${ }^{2}$ & -2.42 & 0.84 & -0.19 & -2.89 & $<0.01$ & $-4.07 ;-0.77$ \\
\hline Smoker $^{2}$ & -5.72 & 1.67 & -0.22 & -3.42 & $<0.01$ & $-9.02 ;-2.42$ \\
\hline $\begin{array}{l}\text { Medical comorbidity: Diseases of the } \\
\text { cardiovascular system (e.g., coronary heart } \\
\text { disease and high blood pressure) }\end{array}$ & 7.76 & 2.31 & 0.22 & 3.36 & $<0.01$ & $3.21 ; 12.31$ \\
\hline
\end{tabular}

$\mathrm{B}=$ unstandardized beta coefficient; $\mathrm{SE}=$ standard error; $\beta=$ standardized beta coefficient; $\mathrm{p}=p$-value; CI: confidence interval; significant results are highlighted; ${ }^{1} 1=$ female; $2=$ male; $3=$ third gender ${ }^{2} 1=$ yes; $2=$ no; ${ }^{3} 0=$ not quoted; $1=$ quoted. 


\section{Discussion}

The first verified COVID-19 infection was reported in Bavaria, Germany, in the early months of 2020 [19]. Fast adjustment processes were implemented by the German healthcare facilities, and rapid efforts were made to respond to the outbreak. This crisis had an inescapable impact on healthcare societies and educational institutions across the country $[20,21]$. The dental society is among the most affected by the pandemic, both internationally and within Germany, due to a variety of reasons impacting its members' psychological well-being and financial security during the crisis and its associated lockdowns [6]. So far, this survey is the first to assess the mental effect of the COVID-19 outbreak on dental students in Germany, countrywide, by the DASS-21 and IES-R survey tools as major screening instruments used globally for healthcare workers and student psychological evaluations.

In this study, 211 dental students participated nationwide, through the web-based survey link. As they finished the online survey, the study population displayed a significant sample size, representing Germany's dental students in the study. In comparison to prior surveys on dental students in Germany (Table 1), the respondents' sociodemographic data revealed a similar gender distribution, with larger percentages of female students [15]. Unsurprisingly, all of the survey contributors presented an age less than 50 years (Table 1), corresponding to the described typical age (20-50 years) among medical university students in Germany [22]. Consequently, the total study population did not express previously described age-related COVID-19 risk factors [6]. Furthermore, the survey participants reported a smoking rate of nearly $6 \%$ (Table 1 ), similar to the conveyed outcomes of dental professionals in Germany (5-8\%), in addition to other oral health societies globally [15]. Cardiovascular diseases displayed the major systemic diseases among a small group of the participating students (Table 1), which is consistent with the results stated by former reports on Germany's population [23].

In the present survey, dental students showed an overall normal or mild psychological impact of the pandemic on anxiety, stress, depression, intrusion, hyperarousal and avoidance, as assessed by the explained survey instruments. This displays a discrepancy to the outcomes of similar studies on healthcare professionals and dental or medical students in other non-European countries, which showed higher levels of psychological distress $[4,6,24-28]$, and could represent the psychological significance of Germany's claimed success in containing COVID-19 infection rates through introducing new safety regulations in health and educational institutions, stabilizing its population's financial situation throughout the crisis, and communicating the reasons for its emergency policies [6,29-31]. Furthermore, several variables distressing students in German universities throughout the outbreak appear to actively participate in the development of stress-, anxiety-, depressionand PTSD-associated symptoms. Similar to previous reports on German dentists [6], the female students in the current survey presented significantly higher anxiety and stress, as well as IES-R subscales (Tables 4 and 5) than the male participants. This situation is explained by earlier findings that females are statistically more likely than men to acquire mental distress, depressive symptoms, and PTSD signs as adults $[29,30]$. This variance between the two genders was described as a result of different thought control approaches during difficult life situations, and discrepant metacognitive beliefs among males and females, promoting an intensified mental effect among the latter ones [30]. While only $0.5 \%$ of the study sample characterized third-gender members of the dental students (Table 1), the single respondent showed intense psychological distress compared to other genders (Tables 4 and 5). This outcome also approves the results seen among third-gender German dentists [6] and reported social tensions, leading to mental health concerns among this part of the society [32]. The current investigation further reported that aspects among the participants, such as being single and having no child responsibilities, were linked to healthier mental outcomes than being in a marriage relationship or raising children (Tables 4 and 5). This consequence was observed similarly among the German population during the COVID-19 crisis, as individuals with no relationship status had a significantly more stable mental health than married couples or individuals living with a partner, specif- 
ically in cases with poor relationship quality [33]. Amongst multiple factors, the economic strain created by the COVID-19 lockdowns might be closely related to a decline in the marital and partnership quality of the affected individuals [34]. Moreover, previous studies on parents' mental health have described how early parenthood often promotes psychological distress and depression [35], which can be associated with the students' in early paternity and motherhood.

Systemic disorders have been previously linked to the higher morbidity and mortality rates of the affected patients during the pandemic [36,37]. As a result, those who have been afflicted conveyed more psychological distress [1]. In the current survey, dental students in Germany have exhibited a similar outcome during the COVID-19 pandemic, particularly among vascular diseases (Tables 4 and 5). This also confirms earlier results reported on German dentists, which have displayed cardiovascular disease as one of the significant factors of mental decline during the pandemic [6]. Indeed, underlying cardiovascular comorbidities are associated with high mortality rates of COVID-19 patients. Moreover, a COVID-19 infection can even induce or deteriorate cardiovascular conditions, such as myocardial infarctions, arrhythmias and venous thromboembolism [38,39]. Hypertension was furthermore described to have the highest prevalence as a comorbidity in COVID-19 patients [40], creating an obvious stress factor for cardiovascular patients [41]. Another comorbidity increasing the risk of COVID-19-associated complications is pulmonary disease [42]. As reported previously, patients having pulmonary conditions presented high incidences of depression, COVID-19-related stress, post-traumatic symptoms, as well as insomnia during the lockdown [43]. Furthermore, as smoking is considered a main risk factor for malignant and nonmalignant conditions of the respiratory tract [44,45], significantly higher mental health distress was noted among the smoking participants of the current survey (Tables 4-7). This conforms to multiple studies describing the higher mental distress among smokers during the pandemic. These reports even observed higher rates of smoking among the affected individuals, leading to a vicious cycle of mental health deterioration and habitual smoking [46-49].

Having to face the unexpected consequences during the pandemic, students, predominantly in the medical field, were among the most affected groups globally, showing increased levels of stress, anxiety, and depression [50-52]. With elevated financial insecurity after losing employment, besides the anxiety about the digitalized home-schooling or delayed graduation, students had numerous difficulties getting through the lockdown and the imposed regulations [51,53]. This outcome was also observable among dental students in Germany, as the respondents that considered COVID-19 as threatening their financial stability had significantly higher scores in the current survey (Tables 4-7). However, this financial pressure might show variances between different German states and regions. Recent studies described the socioeconomic disparities within the German population, based on the cities and living areas [54]. On the other hand, due to the predominantly government- and tax-funded dental and medical education and its services in German universities, and the very similar life standards among the majority of German university students, this socioeconomic discrepancy might be less significant on the student level $[15,55]$. As reported previously, severe restrictions were additionally enforced, particularly on the practical training component of undergraduate dental medical training, creating new challenges for the students and universities in adapting to the new digital platforms and education techniques [20,21]. Moreover, many medical and dental students from German universities displayed higher stress aspects during the pandemic, in terms of being worried about the continuation or quality of their education [56,57]. Interestingly, some investigations have observed an enhancement of students' learning performance during the COVID-19 lockdowns [58]. This was further explained, as students may be driven by their inherent responsibility in confusing situations to contribute as much as they can to solve the difficulties that education is experiencing during the pandemic. This would lead different students to find different motivations to guarantee good and safe progress in this academic year, despite the outbreak [58]. 
Multiple linear regression tests were performed to determine independent outcomes of the significant analyzed factors of the pandemic as a financial risk, participant gender, smoking status, and systemic comorbidities on the survey instrument scores. Female gender, being a smoker or cardiovascular patient, and seeing the pandemic as an economic burden were independently linked with worse psychological outcomes (Tables 4-7), emphasizing these aspects as the most effective on dental students' psychological stability in Germany throughout the COVID-19 pandemic.

\section{Limitations}

To date, this is the first study to examine the mental effect of the COVID-19 pandemic on dental students countrywide in Germany, by using the DASS-21 and IES-R survey instruments. Yet, some limiting aspects to the survey have to be described. Firstly, the study is limited, as it is cross-sectional and has no longitudinal follow-up parts. Our current outcomes of dental students cannot be ascribed entirely to the observed aspects and socio-environmental information. Unrecorded factors (as the exact financial status) could be an essential factor in changing some survey interpretations.

Furthermore, the survey participation was performed during several months. Due to the high sensitivity of the COVID-19 crisis and the rapid fluctuations in the infection rates and applied protocols, such changes could impact the results described by the study participants as well. Additionally, having a voluntary study might produce an effect of selection bias amongst participants. Lastly, to reduce face-to-face contact and maximize the participation, an online self-report questionnaire was applied, to evaluate the psychological symptoms without a diagnostic evaluation by mental health specialists. An addition of a clinical assessment by professionals of psychology would undoubtedly add an important feature to the survey. Notwithstanding the above limitations, the displayed conclusions of this study present essential and unique data on the psychological effect of the ongoing pandemic on dental students in German universities.

\section{Conclusions}

Dental students' mental health is imperative for their educational and behavioral development during the COVID-19 crisis, and their subsequent careers. Our conclusions exhibited that female gender, being chronically ill with cardiovascular conditions, habitually smoking, and seeing COVID-19 as a financial risk are statistically significant aspects leading to a psychological decline among dental students across Germany during the pandemic, and these factors increased IES-R and DASS-21 outcomes. Examining such factors closely could support Germany's educational and health institutions to adjust the implementation of required actions to minimize the psychological harm of the outbreak on Germany's future dentists.

Author Contributions: Conceptualization, M.M., J.C.; data curation, J.C.; investigation, J.C.; M.M. and S.A.; methodology, M.M. and J.C.; project administration, M.M.; software, J.C.; supervision, C.D.; validation, M.M.; writing—original draft, M.M.; writing—review and editing, M.M., J.C., S.A., and C.D. All authors have read and agreed to the published version of the manuscript.

Funding: This research received no external funding.

Institutional Review Board Statement: The study was conducted according to the guidelines of the Declaration of Helsinki and approved by the University of Kiel (D452/18).

Informed Consent Statement: Informed consent was obtained from all subjects involved in the study.

Data Availability Statement: The data presented in this study are available on request from the corresponding author.

Acknowledgments: The authors would like to thank every dental student who participated in this study, and all institutions and individuals that helped distribute this survey among the students in Germany. The authors would also like to thank the state of Schleswig-Holstein and the University of Kiel for the financial support through the Open-Access Funds.

Conflicts of Interest: The authors declare no conflict of interest. 


\section{References}

1. Wang, C.; Pan, R.; Wan, X.; Tan, Y.; Xu, L.; Ho, C.S.; Ho, R.C. Immediate Psychological Responses and Associated Factors during the Initial Stage of the 2019 Coronavirus Disease (COVID-19) Epidemic among the General Population in China. Int. J. Environ. Res. Public Health 2020, 17, 1729. [CrossRef] [PubMed]

2. Ali, S.; Maguire, S.; Marks, E.; Doyle, M.; Sheehy, C. Psychological impact of the COVID-19 pandemic on healthcare workers at acute hospital settings in the South-East of Ireland: An observational cohort multicentre study. BMJ Open 2020, 10, e042930. [CrossRef] [PubMed]

3. Mehta, S.; Machado, F.; Kwizera, A.; Papazian, L.; Moss, M.; Azoulay, É.; Herridge, M. COVID-19: A heavy toll on health-care workers. Lancet Respir. Med. 2021, 9, 226-228. [CrossRef]

4. Hakami, Z.; Khanagar, S.B.; Vishwanathaiah, S.; Hakami, A.; Bokhari, A.M.; Jabali, A.H.; Alasmari, D.; Aldrees, A.M. Psychological impact of the coronavirus disease 2019 (COVID-19) pandemic on dental students: A nationwide study. J. Dent. Educ. 2020, 85, 494-503. [CrossRef]

5. Uhlen, M.M.; Ansteinsson, V.E.; Stangvaltaite-Mouhat, L.; Korzeniewska, L.; Skudutyte-Rysstad, R.; Shabestari, M.; Mdala, I.; Hovden, E.A.S. Psychological impact of the COVID-19 pandemic on dental health personnel in Norway. BMC Health Serv. Res. 2021, 21, 420. [CrossRef]

6. Mekhemar, M.; Attia, S.; Dörfer, C.; Conrad, J. The Psychological Impact of the COVID-19 Pandemic on Dentists in Germany. J. Clin. Med. 2021, 10, 1008. [CrossRef]

7. Proffitt, E. What will be the new normal for the dental industry? Br. Dent. J. 2020, 228, 678-680. [CrossRef]

8. Coulthard, P.; Thomson, P.; Dave, M.; Coulthard, F.P.; Seoudi, N.; Hill, M. The COVID-19 pandemic and dentistry: The clinical, legal and economic consequences-Part 1: Clinical. Br. Dent. J. 2020, 229, 743-747. [CrossRef]

9. Yildirim, T.T.; Atas, O. The evaluation of psychological state of dental students during the COVID-19 pandemic. Braz. Oral Res. 2021, 35, e069. [CrossRef]

10. Santos-Hövener, C.; Busch, M.A.; Koschollek, C.; Schlaud, M.; Hoebel, J.; Hoffmann, R.; Wilking, H.; Haller, S.; Allenj, J.; Wernitz, J.; et al. Seroepidemiological study on the spread of SARS-CoV-2 in populations in especially affected areas in Germany-Study protocol of the CORONA-MONITORING lokal study. J. Health Monit. 2020, 1-15. [CrossRef]

11. Böttcher, S.; Oh, D.-Y.; Staat, D.; Stern, D.; Albrecht, S.; Willrich, N.; Zacher, B.; Mielke, M.; Rexroth, U.; Hamouda, O.; et al. Erfassung der SARS-CoV-2-Testzahlen in Deutschland (Stand 2.12.2020). Epid Bull 2020, 49, 14-20. [CrossRef]

12. Schäfer, M.; Stark, B.; Werner, A.M.; Tibubos, A.N.; Reichel, J.L.; Pfirrmann, D.; Edelmann, D.; Heller, S.; Mülder, L.M.; Rigotti, T.; et al. Health Information Seeking Among University Students Before and During the Corona Crisis-Findings from Germany. Front. Public Health 2021, 8, 616603. [CrossRef]

13. Goldstein, E.; Lipsitch, M. Temporal rise in the proportion of younger adults and older adolescents among coronavirus disease (COVID-19) cases following the introduction of physical distancing measures, Germany, March to April 2020. Euro Surveill. 2020, 25. [CrossRef]

14. Ziller, S.; Eaton, K.E.; Widström, E. The healthcare system and the provision of oral healthcare in European Union member states. Part 1: Germany. Br. Dent. J. 2015, 218, 239-244. [CrossRef]

15. Mekhemar, M.; Conrad, J.; Attia, S.; Dorfer, C. Oral Health Attitudes among Preclinical and Clinical Dental Students in Germany. Int. J. Environ. Res. Public Health 2020, 17, 4253. [CrossRef]

16. Knipe, D.; Maughan, C.; Gilbert, J.; Dymock, D.; Moran, P.; Gunnell, D. Mental health in medical, dentistry and veterinary students: Cross-sectional online survey. BJPsych Open 2018, 4, 441-446. [CrossRef]

17. Bono, R.; Blanca, M.J.; Arnau, J.; Gómez-Benito, J. Non-normal Distributions Commonly Used in Health, Education, and Social Sciences: A Systematic Review. Front. Psychol. 2017, 8, 1602. [CrossRef]

18. Habeck, C.G.; Brickman, A.M. A common statistical misunderstanding in Psychology and Neuroscience: Do we need normally distributed independent or dependent variables for linear regression to work? bioRxiv 2018, 305946. [CrossRef]

19. Böhmer, M.M.; Buchholz, U.; Corman, V.M.; Hoch, M.; Katz, K.; Marosevic, D.V.; Böhm, S.; Woudenberg, T.; Ackermann, N.; Konrad, R.; et al. Investigation of a COVID-19 outbreak in Germany resulting from a single travel-associated primary case: A case series. Lancet Infect. Dis. 2020, 20, 920-928. [CrossRef]

20. Loda, T.; Löffler, T.; Erschens, R.; Zipfel, S.; Herrmann-Werner, A. Medical education in times of COVID-19: German students' expectations-A cross-sectional study. PLoS ONE 2020, 15, e0241660. [CrossRef]

21. Röhle, A.; Horneff, H.; Willemer, M.C. Practical teaching in undergraduate human and dental medical training during the COVID-19 crisis. Report on the COVID-19-related transformation of peer-based teaching in the Skills Lab using an Inverted Classroom Model. GMS J. Med. Educ 2021, 38. [CrossRef]

22. Chenot, J.-F. Undergraduate medical education in Germany. Ger. Med. Sci. 2009, 7. [CrossRef]

23. Plass, D.; Vos, T.; Hornberg, C.; Scheidt-Nave, C.; Zeeb, H.; Krämer, A. Trends in disease burden in Germany: Results, implications and limitations of the Global Burden of Disease study. Dtsch. Arztebl. Int. 2014, 111, 629-638. [CrossRef]

24. Bizri M, Kassir G, Tamim H, Kobeissy F, Hayek SE. Psychological distress experienced by physicians and nurses at a tertiary care center in Lebanon during the COVID-19 outbreak. J. Health Psychol. 2021, 1359105321991630. [CrossRef]

25. Iosif, L.; Ţâncu, A.M.C.; Didilescu, A.C.; Imre, M.; Gălbinașu, B.M.; Ilinca, R. Self-Perceived Impact of COVID-19 Pandemic by Dental Students in Bucharest. Int. J. Environ. Res. Public Health 2021, 18, 5249. [CrossRef] 
26. Elbay, R.Y.; Kurtulmuş, A.; Arpacioğlu, S.; Karadere, E. Depression, anxiety, stress levels of physicians and associated factors in Covid-19 pandemics. Psychiatry Res. 2020, 290, 113130. [CrossRef]

27. Saraswathi, I.; Saikarthik, J.; Senthil Kumar, K.; Madhan Srinivasan, K.; Ardhanaari, M.; Gunapriya, R. Impact of COVID-19 outbreak on the mental health status of undergraduate medical students in a COVID-19 treating medical college: A prospective longitudinal study. PeerJ 2020, 8, e10164. [CrossRef]

28. Islam, M.S.; Sujan, M.S.H.; Tasnim, R.; Sikder, M.T.; Potenza, M.N.; Van Os, J. Psychological responses during the COVID-19 outbreak among university students in Bangladesh. PLoS ONE 2021, 15, e0245083. [CrossRef]

29. Altemus, M.; Sarvaiya, N.; Neill Epperson, C. Sex differences in anxiety and depression clinical perspectives. Front. Neuroendocrinol. 2014, 35, 320-330. [CrossRef]

30. Bahrami, F.; Yousefi, N. Females are more anxious than males: A metacognitive perspective. Iran. J. Psychiatry Behav. Sci. 2011, 5, 83-90.

31. Hertrampf, K.; Flörke, C.; Conrad, J.; Passia, N.; Kunzendorf, B.; Größner-Schreiber, B.; Dörfer, C.; Kern, M.; Orthuber, W.; Wiltfang, J. COVID-19 pandemic: Infection control in dental health care of infected patients-A state concept. Oral Dis. 2020. [CrossRef] [PubMed]

32. Valentine, S.E.; Shipherd, J.C. A systematic review of social stress and mental health among transgender and gender nonconforming people in the United States. Clin. Psychol. Rev. 2018, 66, 24-38. [CrossRef] [PubMed]

33. Pieh, C.; O Rourke, T.; Budimir, S.; Probst, T. Relationship quality and mental health during COVID-19 lockdown. PLoS ONE 2020, 15, e0238906. [CrossRef] [PubMed]

34. Stanley, S.M.; Markman, H.J. Helping Couples in the Shadow of COVID-19. Fam. Process. 2020, 59, 937-955. [CrossRef]

35. Widarsson, M.; Engström, G.; Rosenblad, A.; Kerstis, B.; Edlund, B.; Lundberg, P. Parental stress in early parenthood among mothers and fathers in Sweden. Scand. J. Caring Sci. 2013, 27, 839-847. [CrossRef]

36. Singh, A.K.; Misra, A. Impact of COVID-19 and comorbidities on health and economics: Focus on developing countries and India. Diabetes Metab. Syndr. 2020, 14, 1625-1630. [CrossRef]

37. Zhou, F.; Yu, T.; Du, R.; Fan, G.; Liu, Y.; Liu, Z.; Xiang, J.; Wang, Y.; Song, B.; Gu, X.; et al. Clinical course and risk factors for mortality of adult inpatients with COVID-19 in Wuhan, China: A retrospective cohort study. Lancet 2020, 395, 1054-1062. [CrossRef]

38. Bae, S.; Kim, S.R.; Kim, M.-N.; Shim, W.J.; Park, S.-M. Impact of cardiovascular disease and risk factors on fatal outcomes in patients with COVID-19 according to age: A systematic review and meta-analysis. Heart 2021, 107, 373. [CrossRef]

39. Nishiga, M.; Wang, D.W.; Han, Y.; Lewis, D.B.; Wu, J.C. COVID-19 and cardiovascular disease: From basic mechanisms to clinical perspectives. Nat. Rev. Cardiol. 2020, 17, 543-558. [CrossRef]

40. Ng, W.H.; Tipih, T.; Makoah, N.A.; Vermeulen, J.-G.; Goedhals, D.; Sempa, J.B.; Burt, F.J.; Taylor, A.; Mahalingam, S. Comorbidities in SARS-CoV-2 Patients: A Systematic Review and Meta-Analysis. mBio 2021, 12, e03647-20. [CrossRef]

41. Mukhtar, S. Are individuals with cardiovascular disease at risk of COVID-19-related mental health problems or individuals with cardiovascular disease at risk of cardiovascular disease-related mental health problems during COVID-19? A psychologicalpsychiatric perspective. Med. Hypotheses 2020, 144, 109919. [CrossRef]

42. Gallay, L.; Uzunhan, Y.; Borie, R.; Lazor, R.; Rigaud, P.; Marchand-Adam, S.; Hirschi, S.; Israel-Biet, D.; Valentin, V.; Cottin, V. Risk Factors for Mortality after COVID-19 in Patients with Preexisting Interstitial Lung Disease. Am. J. Respir. Crit. Care Med. 2021, 203, 245-249. [CrossRef]

43. Pedrozo-Pupo, J.C.; Campo-Arias, A. Depression, perceived stress related to COVID, post-traumatic stress, and insomnia among asthma and COPD patients during the COVID-19 pandemic. Chron. Respir. Dis. 2020, 17, 1479973120962800. [CrossRef]

44. Jiang, C.; Chen, Q.; Xie, M. Smoking increases the risk of infectious diseases: A narrative review. Tob. Induc. Dis. 2020, 18, 60. [CrossRef]

45. West, R. Tobacco smoking: Health impact, prevalence, correlates and interventions. Psychol. Health 2017, 32, 1018-1036. [CrossRef]

46. Tzu-Hsuan Chen, D. The psychosocial impact of the COVID-19 pandemic on changes in smoking behavior: Evidence from a nationwide survey in the UK. Tob. Prev. Cessat. 2020, 6, 59. [CrossRef]

47. Stanton, R.; To, Q.G.; Khalesi, S.; Williams, S.L.; Alley, S.J.; Thwaite, T.L.; Fenning, A.S.; Vandelanotte, C. Depression, Anxiety and Stress during COVID-19: Associations with Changes in Physical Activity, Sleep, Tobacco and Alcohol Use in Australian Adults. Int. J. Environ. Res. Public Health 2020, 17, 4065. [CrossRef]

48. Caponnetto, P.; Inguscio, L.; Saitta, C.; Maglia, M.; Benfatto, F.; Polosa, R. Smoking behavior and psychological dynamics during COVID-19 social distancing and stay-at-home policies: A survey. Health Psychol. Res. 2020, 8, 9124. [CrossRef]

49. Bommele, J.; Hopman, P.; Walters, B.H.; Geboers, C.; Croes, E.; Fong, G.T.; Quah, A.C.K.; Willemsen, M. The double-edged relationship between COVID-19 stress and smoking: Implications for smoking cessation. Tob. Induc. Dis. 2020, 18, 63. [CrossRef]

50. Du, J.; Mayer, G.; Hummel, S.; Oetjen, N.; Gronewold, N.; Zafar, A.; Schultz, J.-H. Mental Health Burden in Different Professions During the Final Stage of the COVID-19 Lockdown in China: Cross-sectional Survey Study. J. Med. Internet Res. 2020, 22, e24240. [CrossRef]

51. Jones, H.E.; Manze, M.; Ngo, V.; Lamberson, P.; Freudenberg, N. The Impact of the COVID-19 Pandemic on College Students' Health and Financial Stability in New York City: Findings from a Population-Based Sample of City University of New York (CUNY) Students. J. Urban Health 2021, 98, 187-196. [CrossRef] [PubMed] 
52. Yang, C.; Chen, A.; Chen, Y. College students' stress and health in the COVID-19 pandemic: The role of academic workload, separation from school, and fears of contagion. PLoS ONE 2021, 16, e0246676. [CrossRef]

53. Aristovnik, A.; Keržič, D.; Ravšelj, D.; Tomaževič, N.; Umek, L. Impacts of the COVID-19 Pandemic on Life of Higher Education Students: A Global Perspective. Sustainability 2020, 12, 8438. [CrossRef]

54. Redler, P.; Wuppermann, A.; Winter, J.; Schwandt, H.; Currie, J. Geographic Inequality in Income and Mortality in Germany. Fisc. Stud. 2021, 42, 147-170. [CrossRef]

55. Hauschildt, K.; Vögtle, E.M.; Gwosc, C. Social and Economic Conditions of Student Life in Europe; Synopsis of Indicators—Eurostudent VI 2016-2018; W. Bertelsmann Verlag: Bielefeld, Germany, 2018.

56. Guse, J.; Heinen, I.; Kurre, J.; Mohr, S.; Bergelt, C. Perception of the study situation and mental burden during the COVID-19 pandemic among undergraduate medical students with and without mentoring. GMS J. Med. Educ. 2020, 37. [CrossRef]

57. O'Byrne, L.; Gavin, B.; McNicholas, F. Medical students and COVID-19: The need for pandemic preparedness. J. Med. Ethics 2020, 46, 623-626. [CrossRef]

58. Gonzalez, T.; de la Rubia, M.A.; Hincz, K.P.; Comas-Lopez, M.; Subirats, L.; Fort, S.; Sacha, G.M. Influence of COVID-19 confinement on students' performance in higher education. PLoS ONE 2020, 15, e0239490. [CrossRef] 